\title{
Vortex Induced Vibration of Subsea Umbilicals: A Case Study of Deep Offshore Nigeria
}

\author{
Ugochukwu Kenneth Udeze, ChinwubaVictor Ossia*
}

Offshore Technology Institute, School of Graduate Studies, University of Port Harcourt, Nigeria

Copyright $\bigcirc 2017$ by authors, all rights reserved. Authors agree that this article remains permanently open access under the terms of the Creative Commons Attribution License 4.0 International License

\begin{abstract}
This study involved using typical Joint North Sea Wave Project (JONSWAP) based Umbilical design of lengths $3044 \mathrm{~m}, 3215 \mathrm{~m}, 4481 \mathrm{~m}$ and $2873 \mathrm{~m}$ clamped to Floating Production Storage and Offloading (FPSO) unit in Deep Offshore Nigeria at water depth of $2000 \mathrm{~m}$. Relevant metOcean data was used to simulate the environmental conditions. The process was simulated using different wave spectra on OrcaFlex software. JONSWAP spectra results for Umbilical-1 to Umbilical-4 at near-surface current (End A: $850 \mathrm{~m})$ showed a Transverse Vortex Force $(T V F)$ range of $0.0115-0.018 \mathrm{kN} / \mathrm{m} ; 0.009-0.0149 \mathrm{kN} / \mathrm{m}$; $0.006-0.014 \mathrm{kN} / \mathrm{m} ; 0.0048-0.009 \mathrm{kN} / \mathrm{m}$, respectively. For Ochi-Hubble spectra, the corresponding TVF range obtained include: $0.004-0.0305 \mathrm{kN} / \mathrm{m} ; 0.004-0.028 \mathrm{kN} / \mathrm{m} ; 0.003-$ $0.027 \mathrm{kN} / \mathrm{m} ; 0.003-0.0232 \mathrm{kN} / \mathrm{m}$, respectively. JONSWAP spectra showed maxima $(t, T V F)$ values of $(0.013,0.018)$; (0.012, 0.0149); $(0.008,0.014)$; $(0.0074,0.009)$ from Umbilical-1 to Umbilical-4, respectively. With the Ochi-Hubble spectra, maxima $(t, T V F)$ values were $(0.018$, $0.0305) ;(0.016,0.028) ;(0.013,0.027) ;(0.0141,0.0232)$ for Umbilical-1 to Umbilical-4, respectively. Hence, maxima ( $t$, $T V F)$ values were greater for Ochi-Hubble than JONSWAP. Simulation plots indicate that adjusting the peakedness parameter $(\gamma)$ of the JONSWAP spectra does not give the required accuracy for this region. Therefore, using a JONSWAP based Umbilical design in Ochi-Hubble environment like Offshore Nigeria shows higher vortex induced vibration (VIV) at near surfaces leading to higher $T V F$, and higher fatigue damage tendency.
\end{abstract}

Keywords Transverse Vortex Force, Ochi-Hubble Spectra, JONSWAP Spectra

\section{Introduction}

The marine umbilical may be defined as a coupling of fluid conduits, electrical and fiber optic cables, which are on their own or in combinations of each other, joined together for flexible purpose. It can also be seen as a bundled assembly of tubing, piping and also electrical conductors in armoured sheath which is installed in internal facility of the subsea production system equipment. Umbilical is an equipment installed on a floating production storage and offloading (FPSO) unit to perform many functions such as carrying an electrical signal, hydraulic fluid, electrical power, with a view to control the subsea production and safety equipment like Christmas tree, subsea valves and manifolds.

Fatigue damage in an umbilical tube can be checked using global dynamic analysis with use of a linear Finite Element (FE) analysis computer program, which includes Flexcom, OrcaFlex, and/or RIFLEX. Global analysis involves simulation of the field conditions in terms of sea state and vessel motion and then doing the calculation of the umbilical dynamic responses to the typical environmental conditions. This global analysis will generate time series of cross section curvatures and tensions for each specified longitudinal position along the Umbilical. Irregular sea state of about 100 / 300 with at least one hour durations are normally sufficient to account for long term statistical subsea field environmental conditions. As offshore oil production moves towards floating production systems in deeper waters, the need for dynamic steel tube umbilical is proportionally on an increase. An umbilical system is subjected to continuously dynamic motion due to water currents, waves, and vessel motions. There will be a sort of decrease in total production if by any chance there is a failure due to Vortex induced vibration (VIV) hence economic consequences. The increasing occurrence of fatigue damage on the umbilical motivates us to better understand the effects of Umbilical global configuration and friction stresses.

Oil / Gas production fields can be found all over the world in different environmental conditions which need tailored equipment. Marine umbilical are therefore production systems tailored to perform specified tasks. Its main function is to provide electric power system, hydraulic power system, as well as control signals to the subsea equipment such as wellhead machinery and remotely operated vehicles (ROVs). When the subsea structure is connected to FPSO, the 
umbilical system is subjected to dynamic loading as a result of environmental conditions. The current findings are that oil and gas extraction takes place at increasing water depth, in a harsh environment. This therefore implies that structural analyses and design are getting more challenging hence the importance of dynamic analyses under VIV.

\subsection{Spectral Wave Models Basic Elements}

To derive spectral models, we will consider two basic elements which involve: high frequency $(H F)$ tail and spectral peakedness.

\section{a The High Frequency $(\mathrm{HF})$ Tail}

The characteristics of the high frequency component of the spectrum are given by an energy balance $(E B)$ for waves created by the local wind fields. A very important term in wind wave generation is known as the equilibrium range. It was believed that the wind blows for a long time or over a long fetch $(L F)$ when wave energy at upper limit is reached, which is called the equilibrium range. Therefore, energy input from the wind is balanced by energy losses due to wave breaking and other frequencies. This equilibrium range was introduced by Philips [1] although it is an ongoing discussion proposed to define the high frequency range. Philips equilibrium range can be found in dynamic analysis as given by equation (1).

$$
S(f) \approx \alpha(2 \pi)^{-5} g^{2} f^{-5}
$$

where $\alpha=7.4 \times 10^{-4}$

However, equilibrium range was actually used by Pierson and Moskowitz at the time they developed the so called $P M$-spectrum for fully developed wind sea [2]. Nevertheless, in Hasselman et al. [3], a formula was used in the JONSWAP spectrum to analyze sea state under fetch limited conditions. However, based on measurement of spectra by Toba [4], theoretical study has shown wave-wave interactions and energy input (EI) mechanisms from wind and wave breaking. The equilibrium range for high frequency was observed by Philips [5] to be given by equation (2).

$$
S(f) \approx u^{*}(2 \pi)^{-4} f^{-4}
$$

The theoretical $H F$ tail value was believed to be the exponent value for wind created by the sea as in this work. Considering measured spectral, the so called exponent has been assumed to be between 4 and 5. But some values outside the range were found in some cases. This may be due to measurement technique, bottom effect, ocean current or other effects.

\section{b Spectral Peakedness}

Energy can follow the equilibrium range for wave spectrum up to the spectra peak, then drops to 0. Formulation of wave spectral model (WSM) developed by Bretschneider [6] was given as equation (3)

$$
S(f)=\alpha(2 \pi)^{-5} g^{2} f^{-5} e^{\left(-0.675\left(1 / T_{m} f\right)^{4}\right)}
$$

Equation (3) at high frequencies agrees with Phillips $1^{\text {st }}$ version of the equilibrium range and confirms $\alpha=$
$7.4 \times 10^{-4}$ which use measured theoretically. In the later experiment, Pierson and Moskowitz came up with another spectrum as in equation (4)

$$
S(f)=\alpha(2 \pi)^{-5} g^{2} f^{-5} e^{\left(-\beta\left(f_{o} / f\right)^{4}\right)}
$$

However, as part of the Joint North Sea Project (JONSWAP), Hasselman et al. [3] developed the JONSWAP spectrum where peak modification or enhancement factor $(E F)$ was introduced to represent fetch limited Wind Sea given as equation (5).

$$
S(f)=\alpha(2 \pi)^{-4} g^{2} f^{-5} e^{\left(-1.25\left(f_{p} / f\right)^{4}\right)} * \gamma^{e^{\left\{\left(\frac{-\left(f-f_{p}\right)^{2}}{2 \sigma^{2} f^{2}}\right)\right\}}}
$$

It was observed that $\alpha$ and $f_{p}$ is dependent on wind speed, fetch and period, so that peakedness parameter can be written as equation (6).

$$
\gamma_{F}\left(f_{n, \gamma}, \sigma\right)=\gamma^{e^{\left\{-\left(\frac{1}{2 \sigma^{2}}\right)\left(f_{n}-1\right)^{2}\right\}}}
$$

Equation (6) represents what is known as JONSWAP peak enhancement function for the adjustment of spectrum peak in other to suit any design model which is only peculiar to JONSWAP. The parameter $\sigma=0.07$ for $f_{n}>1$ and $\sigma=0.09$ for $f_{n}>1$ are normally used. Normalization factor that is related to Pierson and Moskowitz [2] model is of the form in equation (7)

$$
G_{0}=\left\{\left(\frac{1}{M}\right)(N / M)^{-\frac{N-1}{M}} \Gamma\left(\frac{N-1}{M}\right)\right\}^{-1}
$$

where $M=N=4$, it is use when $G_{o}=3.6$

\subsection{D-Peak Wave Spectra}

From elementary physics, it was proved that low frequency waves propagate faster than the generating wind field counterpart to reach those areas that were not influenced directly by the later. The spectrum analysis shows that the swell will add to the locally generated wind sea thereby creating a peak or multiple spectral. Obviously, a sea with different systems will result to different peak frequencies as well as different directions of propagation.

Ochi-Hubble [7], formulated a D-peak spectra model in which the result spectrum was modeled as a summation of two Gamma distribution functions as equation (8).

$$
S(f)=\sum_{j=1}^{2} \frac{H_{s j}{ }^{2} T_{p j}\left(\lambda_{j}+0.25\right)^{\lambda j}}{4 \Gamma\left(\lambda_{j}\right)\left(T_{p j} f\right)^{\left(4 \lambda_{j}+1\right)}} \exp \left\{-\frac{\left(\lambda_{j}+0.25\right)}{\left(T_{p j} f\right)^{4}}\right\}
$$

The three major important parameters are $H_{s}, T_{p}$ and $\lambda$. For analytical purposes, the following recommendations were made for shape factors: $j=1$ for swell sea system and $j=2$ for $W S$ system.

It has been shown that the Ochi - Hubble spectra model is the most probable spectrum which is within $95 \%$ confidence limit for any given value of $H_{s}$.

\subsection{Spectral Model Conventional Analysis}

There are so many parameters spectra models which have 
been considered over the year for single dimension spectral. Some with one peak just as given in the equation (9).

$$
S(f)=P f^{-n} e^{-Q} f^{-m}
$$

The equation (9) is very common for the design of wind and swells according to Massel [8]. So much models was derived from main spectral of different values of the exponent $n$ and $m$. Special additional class is obtained for $n=$ $m=1$. It will yield $P M$-spectrum for $n=5$, Davidan spectrum for $n=5$, Davidan and Massel - spectrum for $n=6$, and $\mathrm{W}$-spectrum when $\mathrm{m}=4$.

One of the most acceptable spectrum models is PM-spectrum. It is used in both medium and very high altitudes. It design for completely developed sea with infinite $4^{\text {th }}$ spectral moment $\mathrm{m}^{4}$ which possesses difficulty in a narrow bandwidth assumptions. In the case of a sea that does not have fully developed range, maybe due to limited fetch, a better model is obtain by modifying this so as to represent narrow bandwidth sea state of that of the North Sea in the JONSWAP design projects in which it is named by Hasselman et al. [3], [9]. Although, this was designed for North Sea, the JONSWAP model can be used anywhere by adjusting its bandwidth to change the peakedness $(\gamma)$. Nevertheless, there is little evidence to back up the claim that if there is a climate change different from that of North Sea, then the JONSWAP model can be used. In other to analyze sea state having many swell separated peaks, many researchers have suggested 2-peak models. These 2-peaked models are normally design by combining two single spectral; in some cases the parameters can be conditioned in order to minimize their number. These models have common characteristics for which they are designed in medium wind sea and high latitudes. The review on the limitation of conventional spectra shape when it comes to swell was done in the report of Mansour and Ertekins [10]. Single peak model is inadequate to be used in West Africa wave models with several swell components since the model must be able to take up to 3-peak or more. Besides, the portioning of the spectra wave component can significantly be improved upon in the area of the accuracy of the computed responses (Quiniou et al. [11]).

\section{Materials and Methods}

\subsection{Umbilical Material Properties}

A typical Umbilical design data with a design life of 20years for an FPSO in Offshore Nigeria was collated. Relevant data below were imputed into OrcaFlex software interface include: Outer diameter nominal $-178 \mathrm{~mm}$, Umbilical Minimum Bending Radius (MBR Operational) $12 \mathrm{~m}$, Bending Stiffness- $2.68 \times 10^{4} \mathrm{Nm}^{2}$, Axial Stiffness$4.62 \times 10^{8} \mathrm{~N}$, Torsional Stiffness $-2.06 \times 10^{4} \mathrm{Nm}^{2}$.

The general design criteria considered were the following environmental data: Design water depth- $2000 \mathrm{~m}$, Seabed Temperature $+4^{\circ} \mathrm{C}$, Sea surface Temperature $+30^{\circ} \mathrm{C}$.

The lengths of the four (4) Umbilicals are: Umbilical-1 3044m, Umbilical-2 - 3215m, Umbilical-3 - 4481m and Umbilical-4 - 2873m.

From design data, buoyancy modules were applied from section length $1379.7 \mathrm{~m}$ to $1479.7 \mathrm{~m}$ for each umbilical. Relevant material data were imputed on the OracaFlex software as shown in the Figure 1.

\subsection{MetOcean Data}

MetOcean data for Offshore Nigeria including water depth, current factor, rotation, near-surfaces and near-bottom current speed and direction were imputed into the OrcaFlex platform for both JONSWAP and Ochi-Hubble wave spectra.

Another metOcean data involving the near-bottom surface current velocity versus simulation time variation showed 0 , $0.103,0.206,0.309,0.412$, and $0.514 \mathrm{~m} / \mathrm{s}$ velocity for $0,2,4$, 6,8 , and 10 s simulation time, respectively

\subsection{Methods}

The Umbilical material characteristics and metOcean data were inputted into the OrcaFlex 8.4a7 [12] software platform and simulated using the Milan wake oscillator model for VIV. Results were obtained for the Transverse Vortex Force (TVF) at different sections for both Ochi-Hubble and JONSWAP wave spectra. 


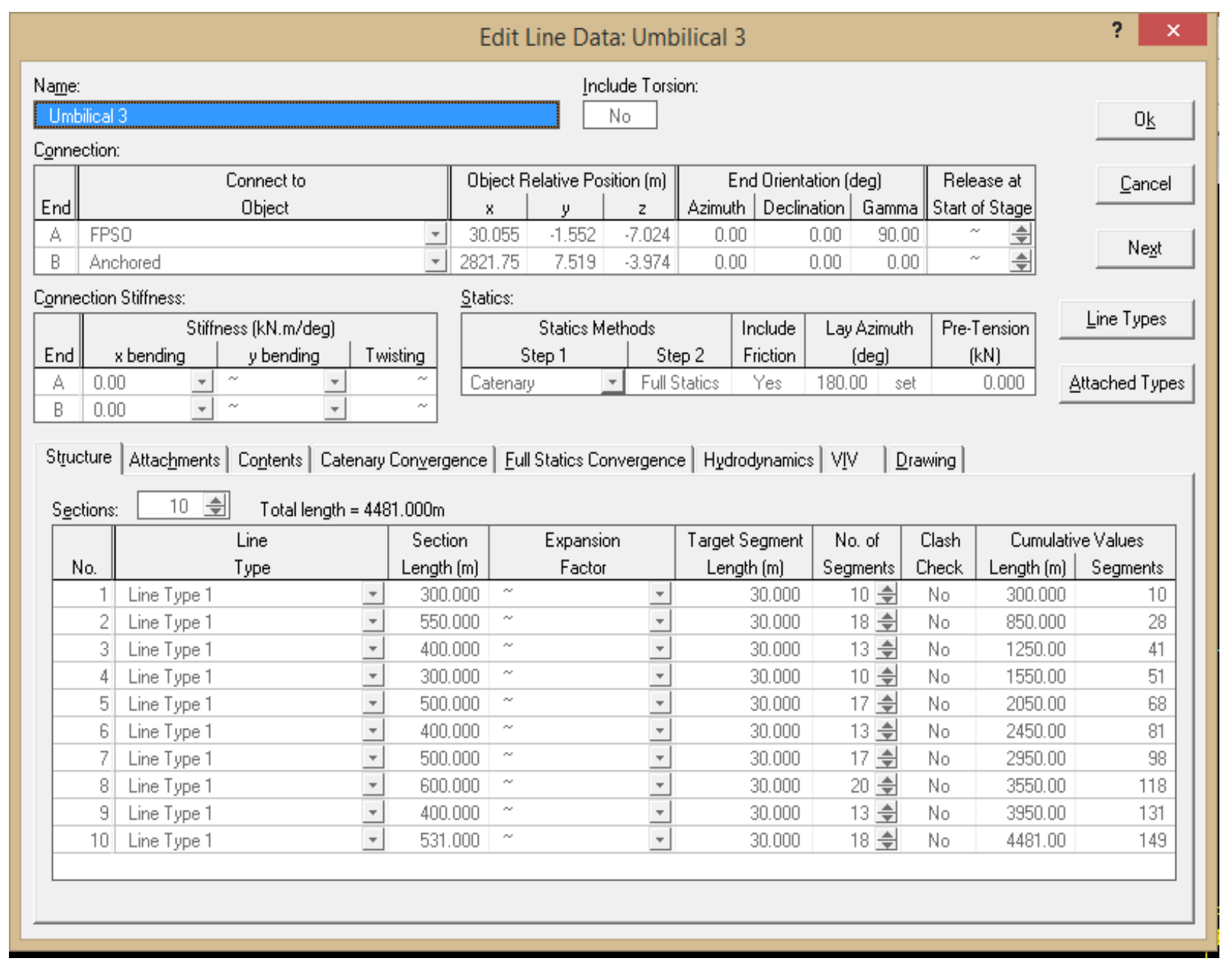

Figure 1. Typical Umbilical Material Description on OrcaFlex Interface

\section{Results and Discussion}

\subsection{JONSWAP Wave Spectra}

Data extracted from Figure 2, Figure 3 and the other metOcean data involving the near-bottom surface current velocity versus simulation time variation were inputted into the environment and variable data section of the OrcaFlex platform. 


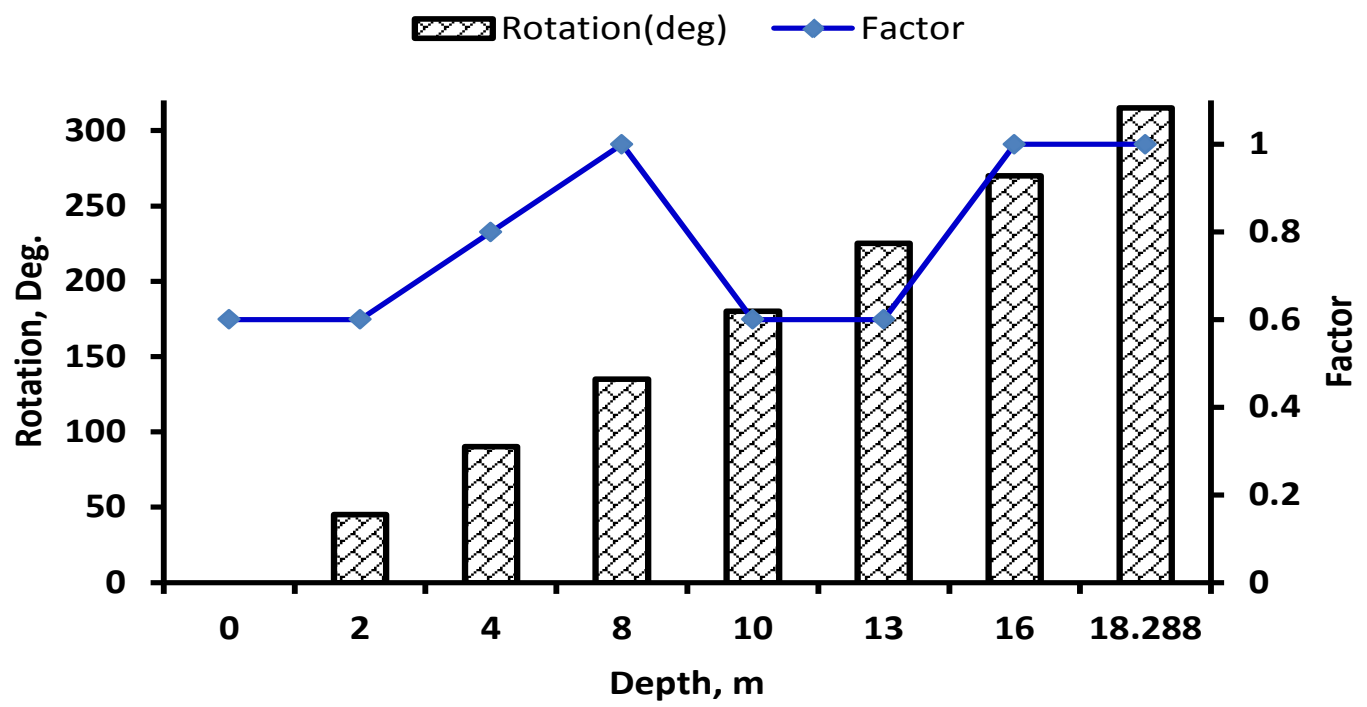

Figure 2. Current Factor and Angle of rotation for various water depths as simulated on OrcaFlex

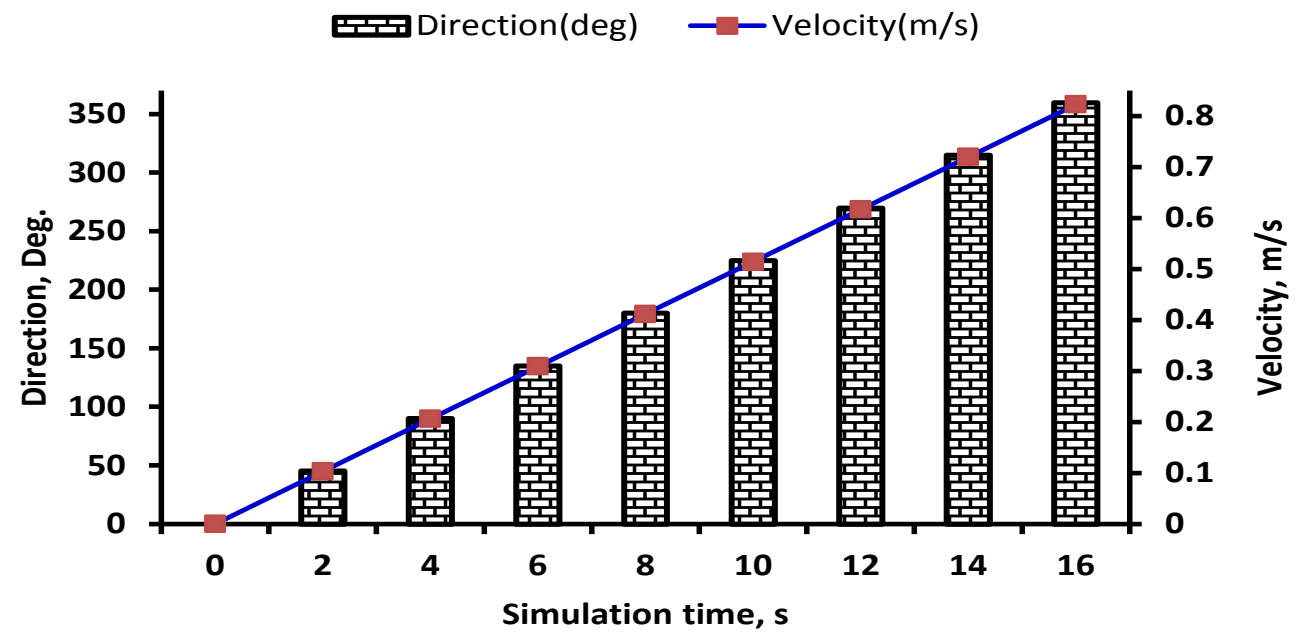

Figure 3. Near Surface Current velocity and Direction versus simulation time

For JONSWAP wave spectra the following results were obtained for TVF; 


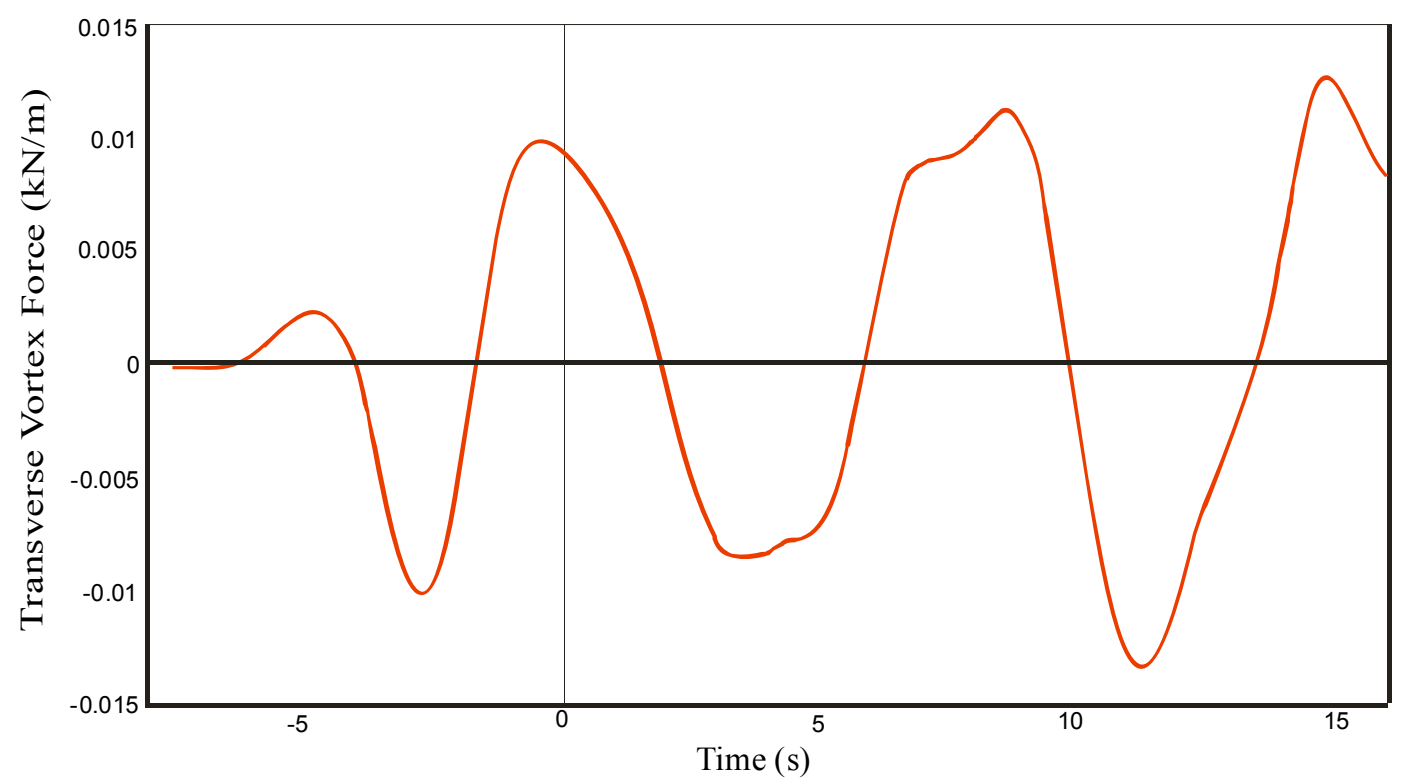

Figure 4. TVF- time graph of Umbilical-1 at 300m (Near Surface Current)

At Umbilical-1 length $300 \mathrm{~m}$, the $T V F$ - time plot showed maxima at $(t, T V F)=(8.0,0.0115) ;(14.1 ; 0.013)$. The highest maximum is $0.013 \mathrm{kN} / \mathrm{m}$. No values exist for the minimum since it is negative. Fluctuations occur at this segment due to instability in current flow and wave spectral properties hence the reason for the graph showing mesokurtic peaks of both double and single nature as well as flat sags and gullies.

Similarly, Umbilical-1 length $600 \mathrm{~m}$ showed maxima at $(t, T V F)=(8.0,0.018) ;(14.6,0.0175)$. The highest maximum is $0.018 \mathrm{kN} / \mathrm{m}$. At this segment some stability exists in the current flow and wave spectral properties, hence the single mesokurtic peaks with sharp gullies. No values exist for minimum since its negative.

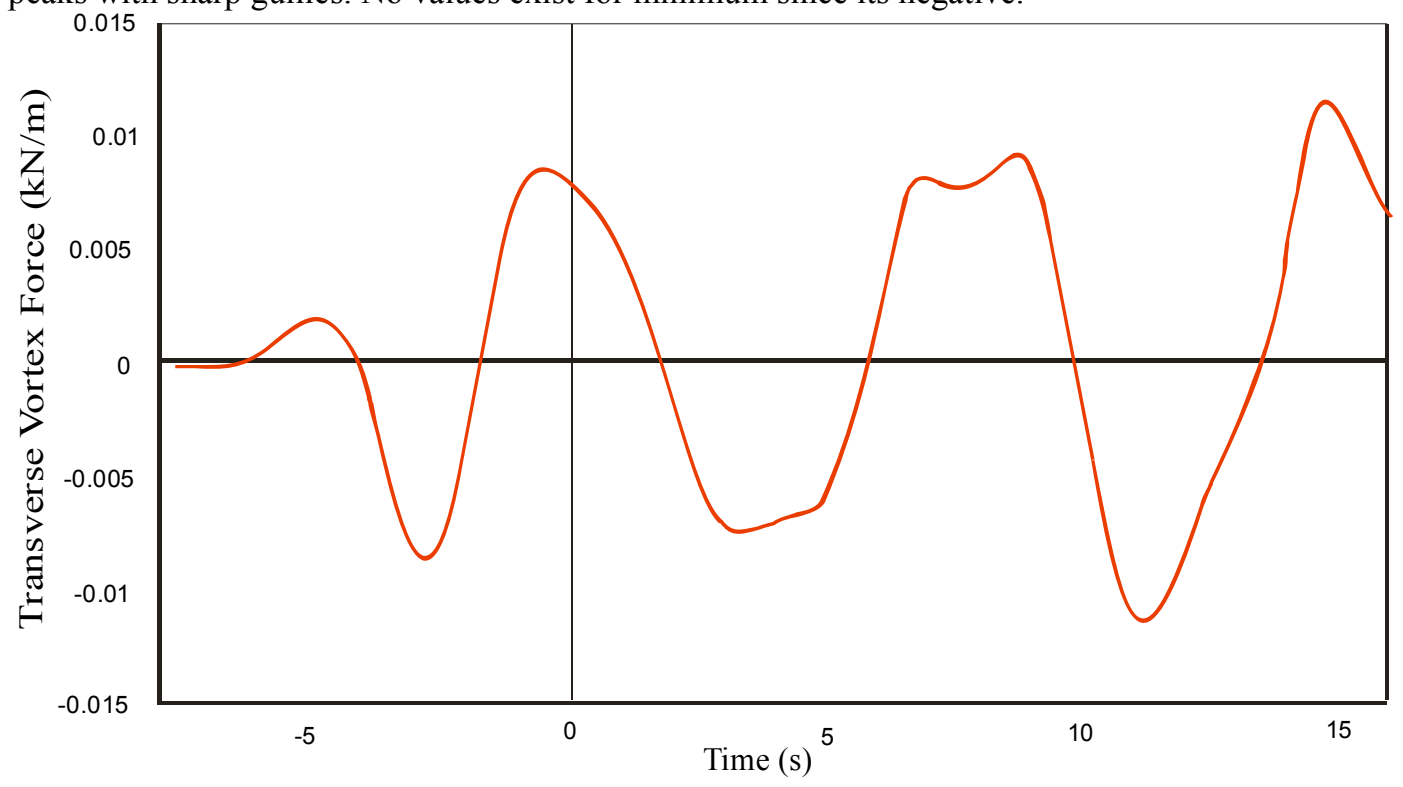

Figure 5. TVF- time graph of Umbilical-2 at $300 \mathrm{~m}$ (Near Surface Current)

Similarly, Umbilical-2 length $600 \mathrm{~m}$ showed maxima at $(t, T V F)=(8.0,0.009) ;(14.3,0.012)$. The highest maxima is 0.012 $\mathrm{kN} / \mathrm{m}$. Fluctuations occur at this segment due to instability in current flow and wave spectral properties hence the reason for the graph showing mesokurtic peaks of both double and single nature as well as flat and gully sags. No minimum values exist.

Also, Umbilical-2 at $600 \mathrm{~m}$ showed maxima $(t, T V F)=(7.6,0.0148) ;(14.2,0.0149)$. The highest maximum is $0.0149 \mathrm{kN} / \mathrm{m}$. At this segment some stability exists in the current flow and wave spectral properties, hence the single mesokurtic peaks with sharp gullies. No values exist for minimum since its negative. 


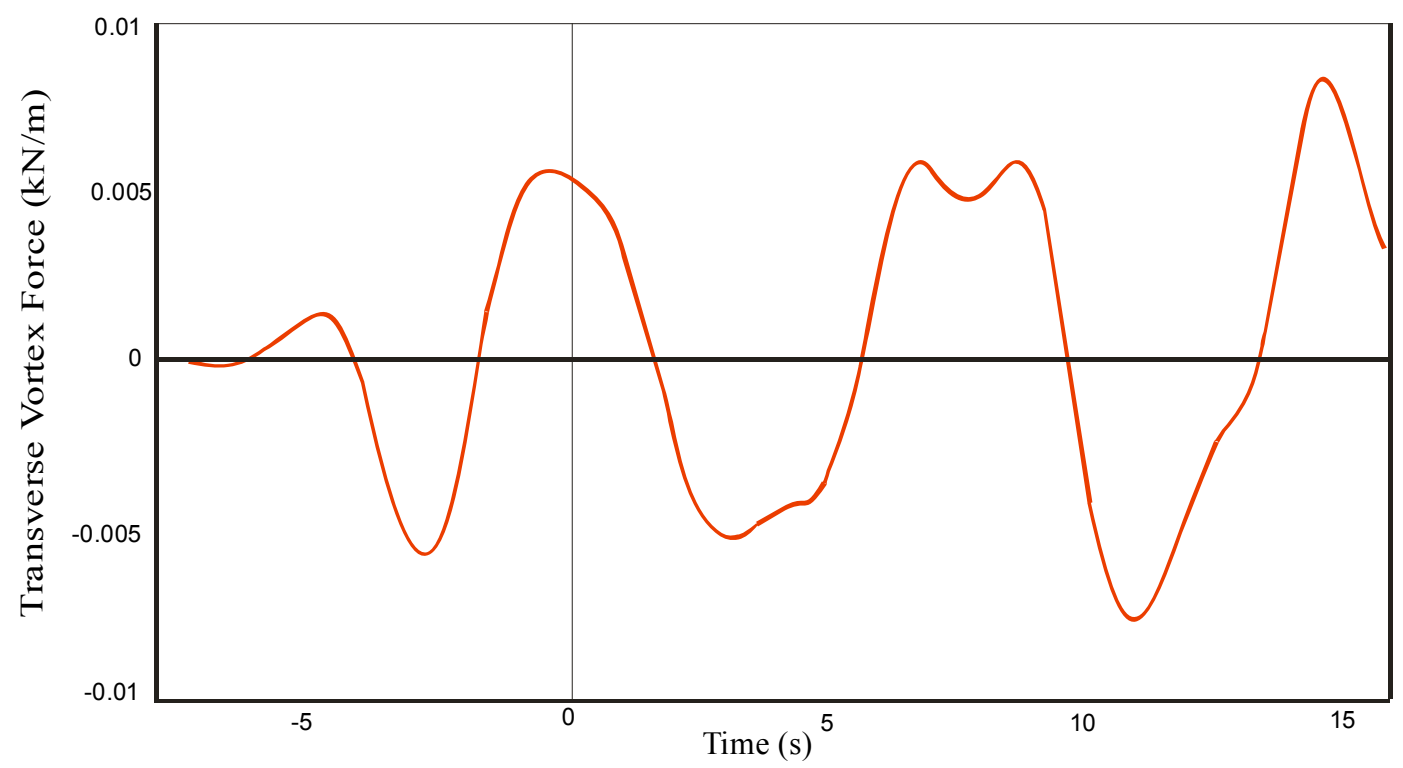

Figure 6. TVF time graph of Umbilical-3 at 300m (Near Surface Current)

Umbilical-3 at $300 \mathrm{~m}$ showed maxima at $(t, T V F)=(7.0,0.006) ;(8.1,0.006) ;(14.5,0.008)$. The highest maxima is $0.008 \mathrm{kN} / \mathrm{m}$. Minimum value is negative. Fluctuations occur at this segment due to instability in current flow and wave spectral properties hence the reason for the graph showing mesokurtic peaks of both double and single nature as well as flat sags and gullies.

Similarly, Umbilical-3 at $850 \mathrm{~m}$ showed maxima $(t, T V F)=(0.3,0.0125) ;(8.4,0.014) ;(14.9,0.012)$. The highest maximum is $0.014 \mathrm{kN} / \mathrm{m}$. No minimum values exist. At this segment some stability exists in the current flow and wave spectral properties, hence the single mesokurtic peaks with sharp gullies.

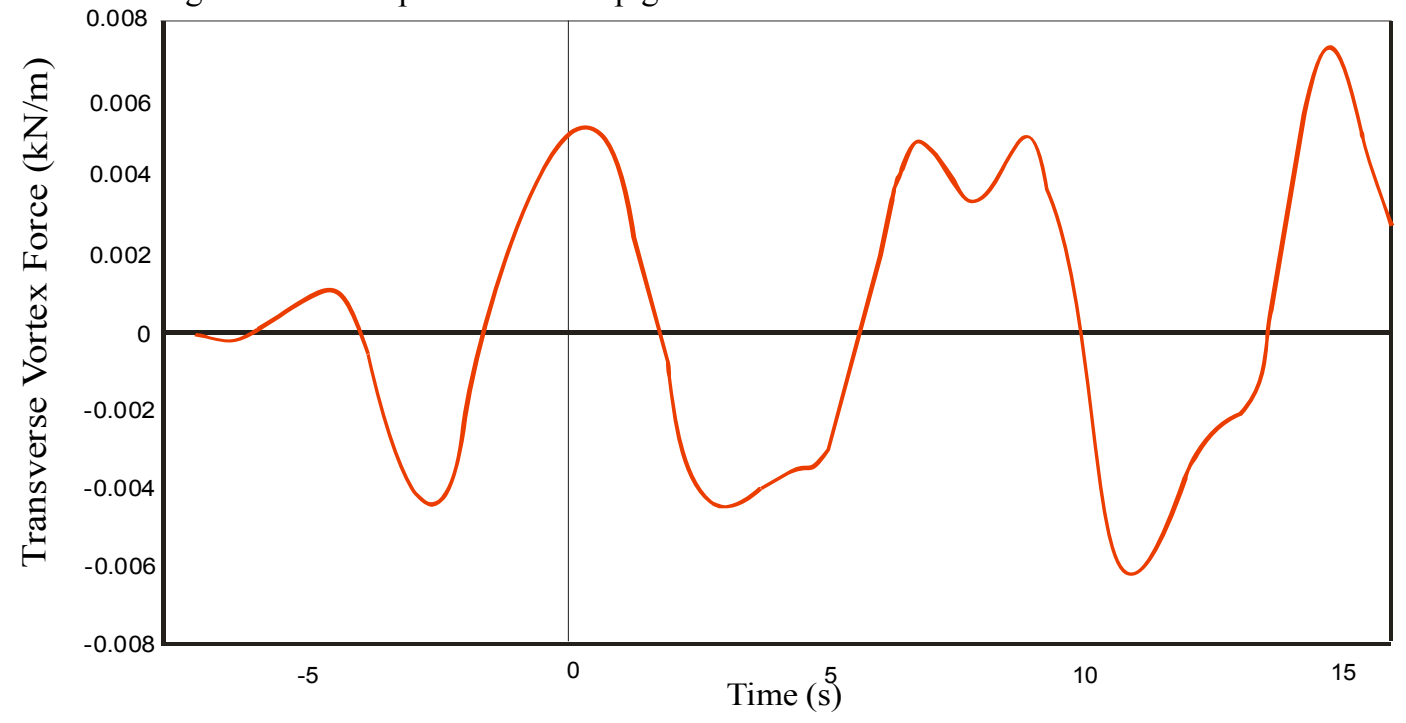

Figure 7. TVF time graph of Umbilical-4 at $300 \mathrm{~m}$ (Near Surface Current)

Umbilical-4 at 300m showed maxima at $(t, T V F)=(0.9,0.0053) ;(6.4,0.0048) ;(8.5,0.0051) ;(14.4,0.0074)$. The highest maximum is $0.0074 \mathrm{kN} / \mathrm{m}$. No minimum values exist. Fluctuations occur at this segment due to instability in current flow and wave spectral properties hence the reason for the graph showing mesokurtic peaks of both double and single nature as well as flat, sags and gullies.

Also, Umbilical-4 at $600 \mathrm{~m}$ showed maxima at $(t, T V F)=(0.5,0.009) ;(8.6,0.0072) ;(14.7,0.0086)$. The highest maximum is $0.009 \mathrm{kN} / \mathrm{m}$. No minimum values exist. At this segment some stability exists in the current flow and wave spectral properties, hence the single mesokurtic peaks with sharp gullies.

For the JONSWAP spectra analysis, the results of Figure 4, 5, 6 and 7 at 300m showed double-peak while Umbilical-1, Umbilical-2, Umbilical-4 at $600 \mathrm{~m}$ and 3 at $850 \mathrm{~m}$, showed single peak. But significantly as we go down in depth for the four umbilicals at $600 \mathrm{~m}$ (Umbilical-1 to Umbilical-3) and $850 \mathrm{~m}$ for Umbilical-4, there is shown a return to single peak which is 
the normal JONSWAP model. This shows that as we go deeper the adjustment of the peakedness parameter to suit Offshore Nigeria Ochi-Hubble environment loses its applicability and tends to revert back to the natural wave spectra of this region.

\subsection{Ochi-Hubble Wave Spectra}

Raw data from Figure 2, Figure 3 and the other metOcean data involving the near-bottom surface current velocity versus simulation time variation were imputed into the environment and variable data section of the OrcaFlex platform. For Ochi-Hubble wave spectra the following results were obtained for $T V F$;

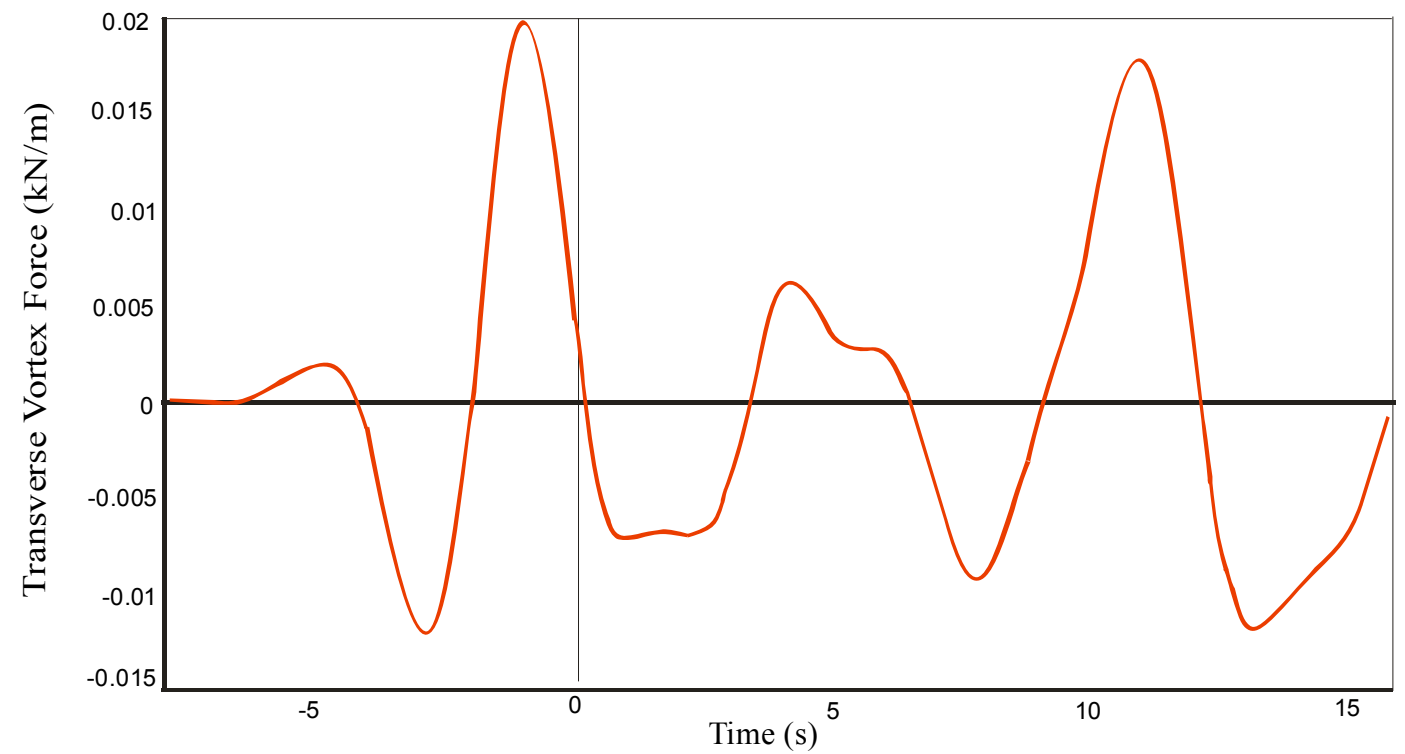

Figure 8. TVF time graph of Umbilical-1 at $300 \mathrm{~m}$ (Near Surface Current)

Umbilical-1 at length $300 \mathrm{~m}$ showed maxima at $(t, T V F)=(4.0,0.006) ;(11.1,0.018)$. The highest maximum is $0.018 \mathrm{kN}$. No minimum value exists. Fluctuations occur at this segment due to instability in current flow and wave spectral properties hence the reason for the graph showing mesokurtic peaks of both double and single nature as well as flat sags and gullies. Similarly, Umbilical-1 at $600 \mathrm{~m}$ showed maxima $(t, T V F)=(3.8,0.004) ;(5.6,0.0075) ;(11.2,0.0305)$. The highest maximum is $0.0305 \mathrm{kN} / \mathrm{m}$. No minimum values exist. Fluctuations occur at this segment due to instability in current flow and wave spectral properties hence the reason for the graph showing mesokurtic peaks of both double and single nature as well as flat sags and gullies.

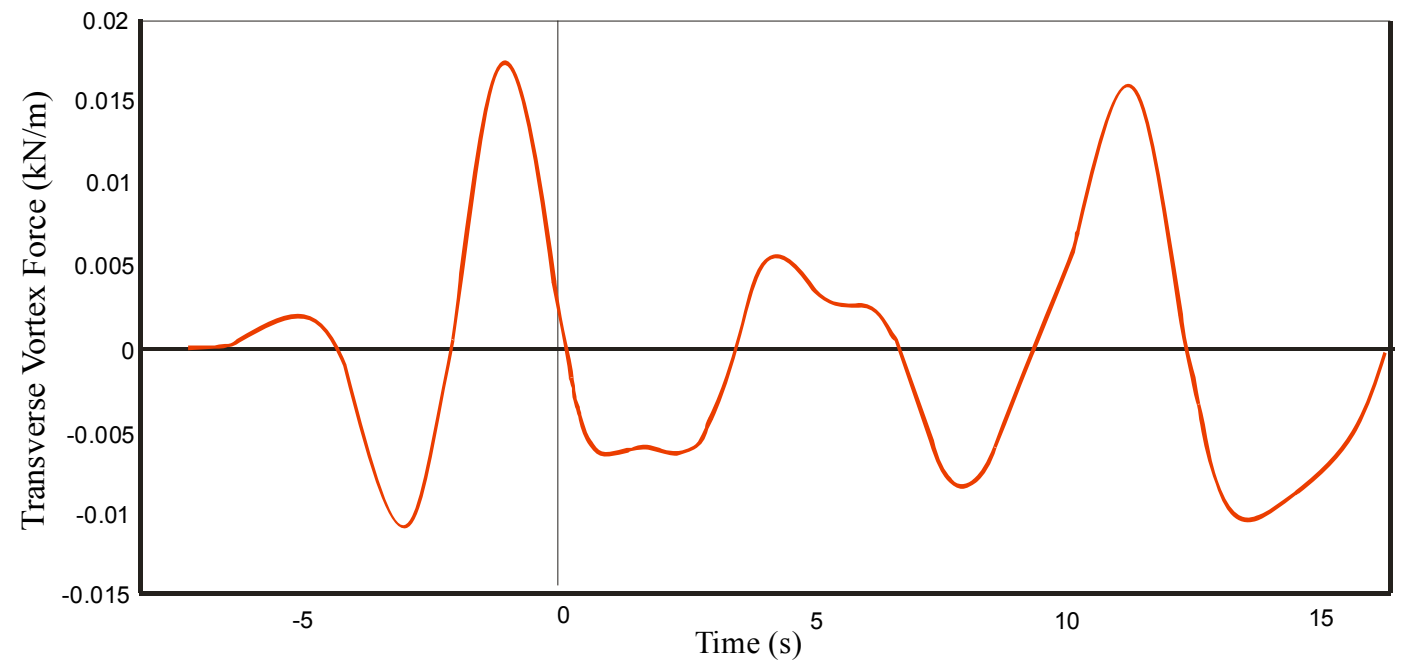

Figure 9. TVF time graph of Umbilical-2 at 300m (Near Surface Current)

Umbilical-2 at length $300 \mathrm{~m}$ showed maxima at Maxima $(t, T V F)=(4.2,0.00508),(12.1,0.016)$. The highest maxima is $0.016 \mathrm{kN} / \mathrm{m}$. No minimum value exists. Fluctuations occur at this segment due to instability in current flow and wave spectral properties hence the reason for the graph showing mesokurtic peaks of both double and single nature as well as flat sags and 
gullies.

While Umbilical-2 at $600 \mathrm{~m}$ showed maxima at $(t, T V F)=(3.7,0.004) ;(5.7,0.007) ;(11.0,0.028)$. The highest maxima is $0.028 \mathrm{kN} / \mathrm{m}$. Fluctuations occur at this segment due to instability in current flow and wave spectral properties hence the reason for the graph showing mesokurtic peaks of both double and single nature as well as flat sags and gullies.

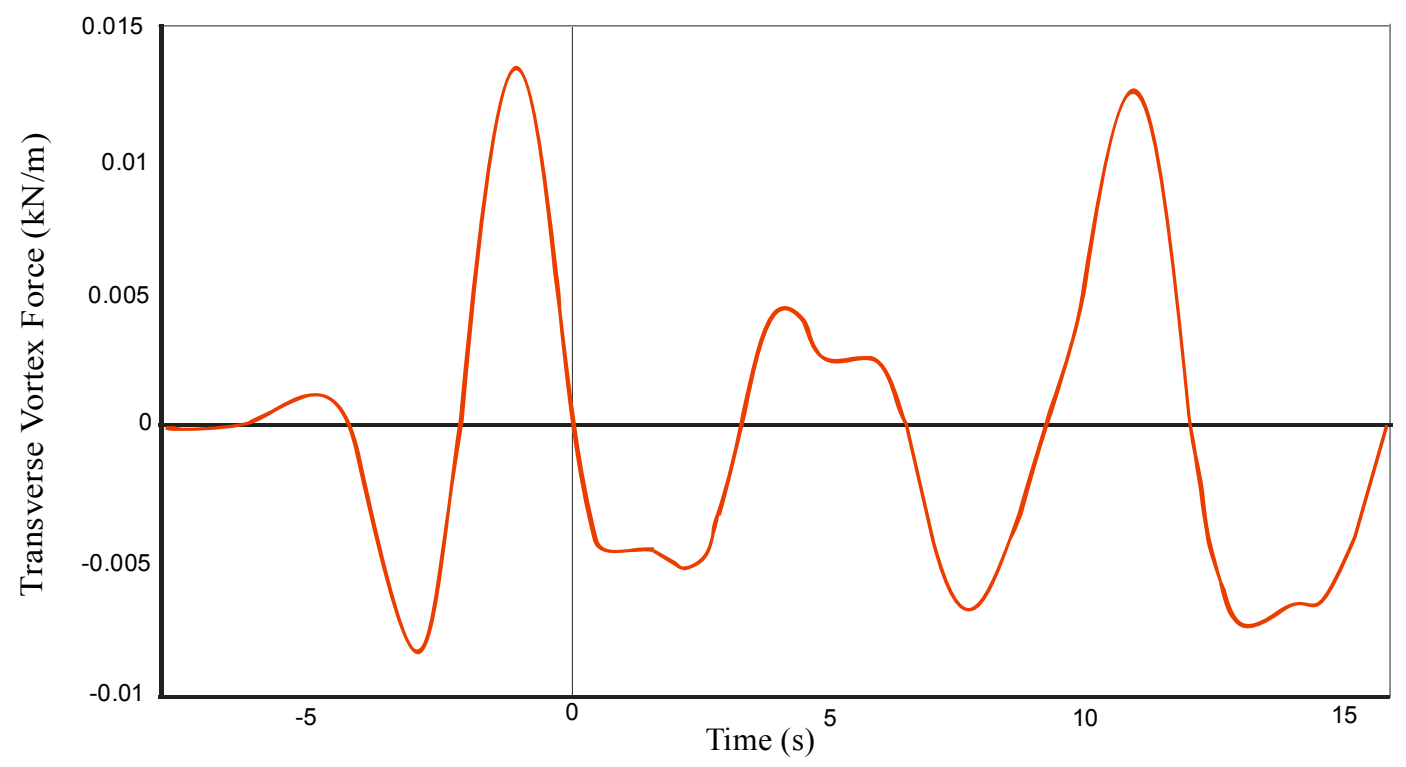

Figure 10. TVF- time graph of Umbilical-3 at 300m (Near Surface Current)

Umbilical-3 at $300 \mathrm{~m}$ showed maxima at $(t, T V F)=(4.0,0.0041) ;(11.5,0.013)$. The highest maxima is $0.013 \mathrm{kN} / \mathrm{m}$. Fluctuations occur at this segment due to instability in current flow and wave spectral properties hence the reason for the graph showing mesokurtic peaks of both double and single nature as well as flat sags and gullies.

Also, Umbilical-3 at $850 \mathrm{~m}$ showed maxima at $(t, T V F)=(3.0,0.003) ;(5.9,0.0074) ;(11.3,0.027)$. The highest maxima is $0.027 \mathrm{kN} / \mathrm{m}$. Fluctuations occur at this segment due to instability in current flow and wave spectral properties hence the reason for the graph showing mesokurtic peaks of both double and single nature as well as gullies.

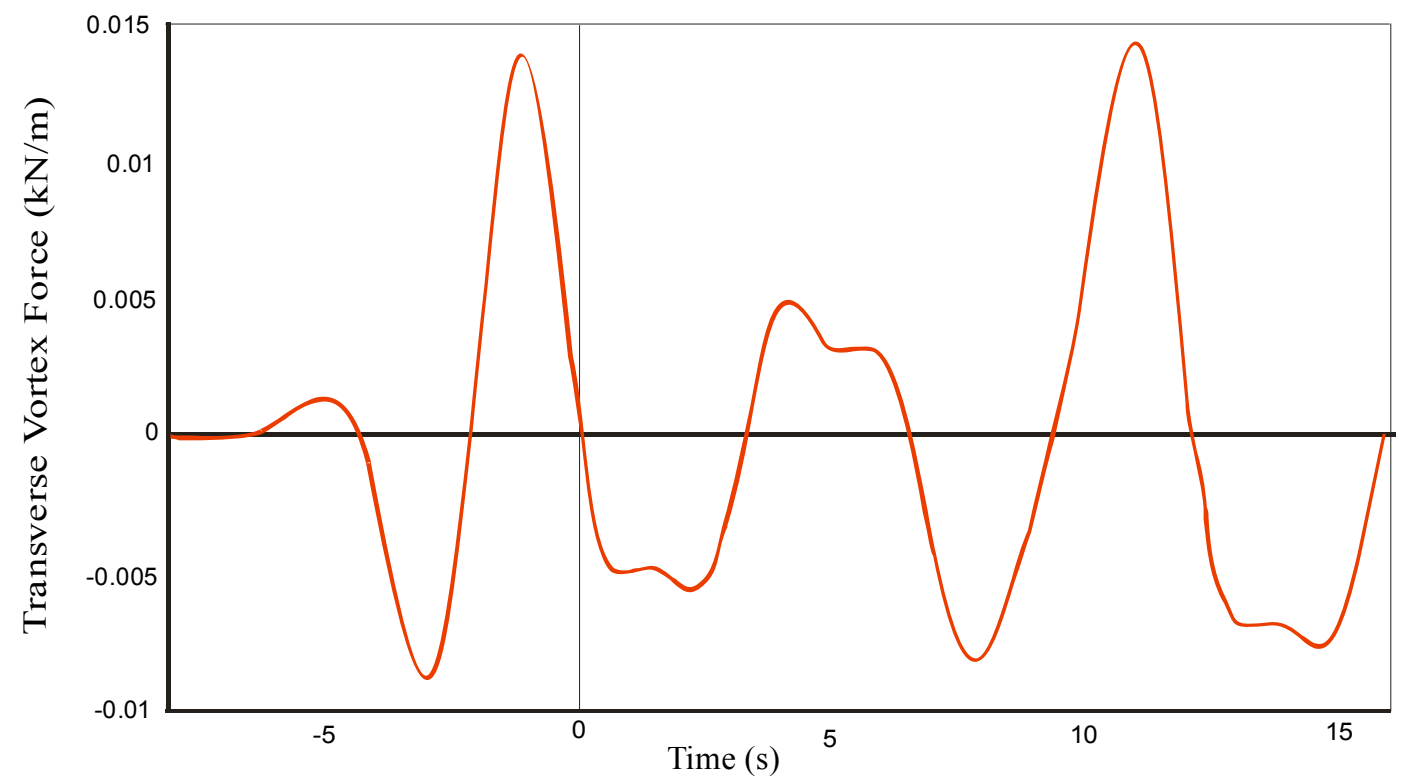

Figure 11. TVF time graph of Umbilical-4 at 300m (Near Surface Current)

Figure 11 showed maxima for Umbilical-4 at 300m. Where $(t, T V F)=(4.1,0.005) ;(6.2,0.0031) ;(11.3,0.0141)$. The highest maxima is $0.0141 \mathrm{kN} / \mathrm{m}$. Fluctuations occur at this segment due to instability in current flow and wave spectral properties hence the reason for the graph showing mesokurtic peaks of both double and single nature as well as flat sags and gullies. 
Similarly, Umbilical-4 at $600 \mathrm{~m}$ showed maxima $(t, T V F)=(3.2,0.003) ;(5.6,0.007) ;(11.1,0.0232)$. The highest maxima is $0.0232 \mathrm{kN} / \mathrm{m}$. Fluctuations occur at this segment due to instability in current flow and wave spectral properties hence the reason for the graph showing mesokurtic peaks of both double and single nature as well as flat sags and gullies.

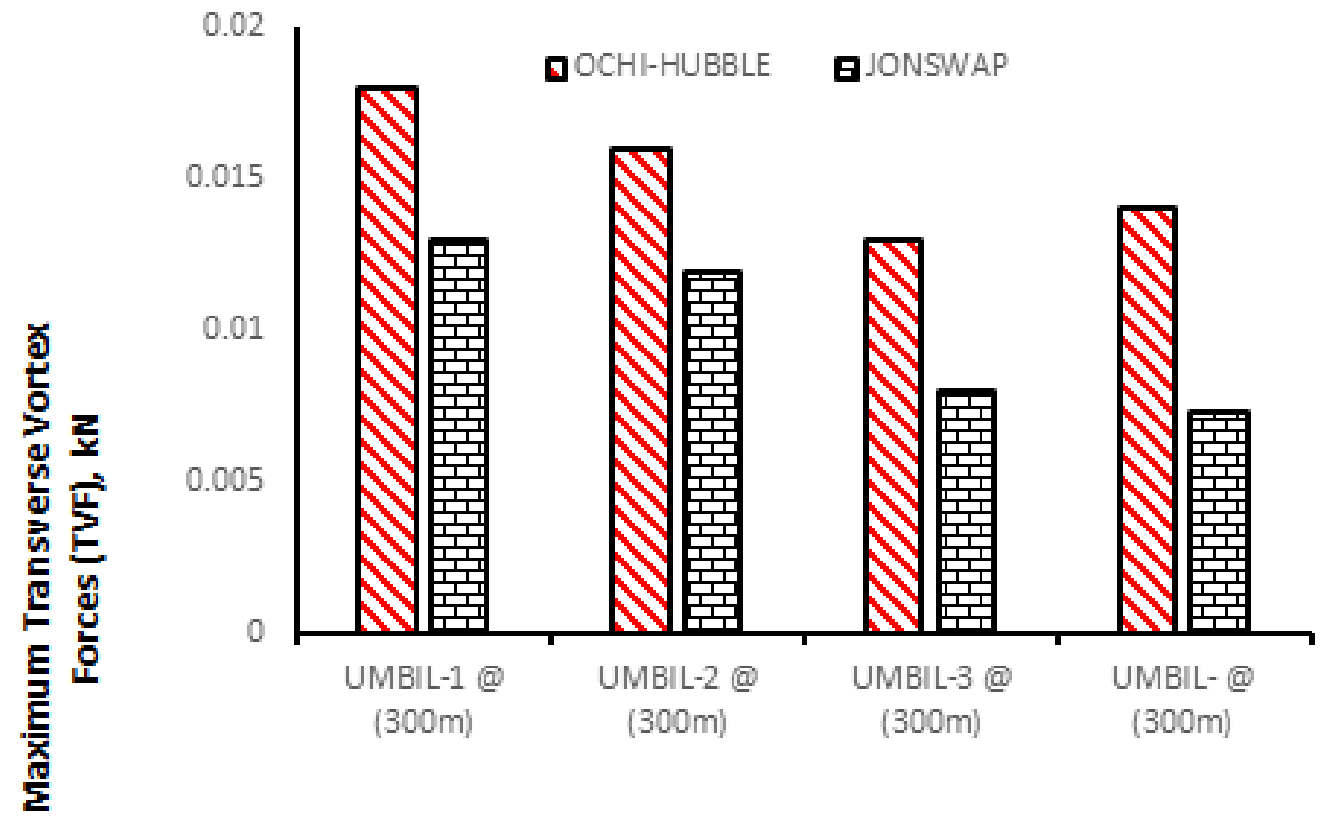

Figure 12. TVF Maxima for Ochi - Hubble and JONSWAP at 300m

Also Figure 12 shows descending order for the heights from Umbilical-1 to 4 at 300m for JONSWAP but for Ochi-Hubble it showed descending heights from Umbilical-1 to Umbilical-3 with Umbilical-4 being the exception as it shows an ascending height.

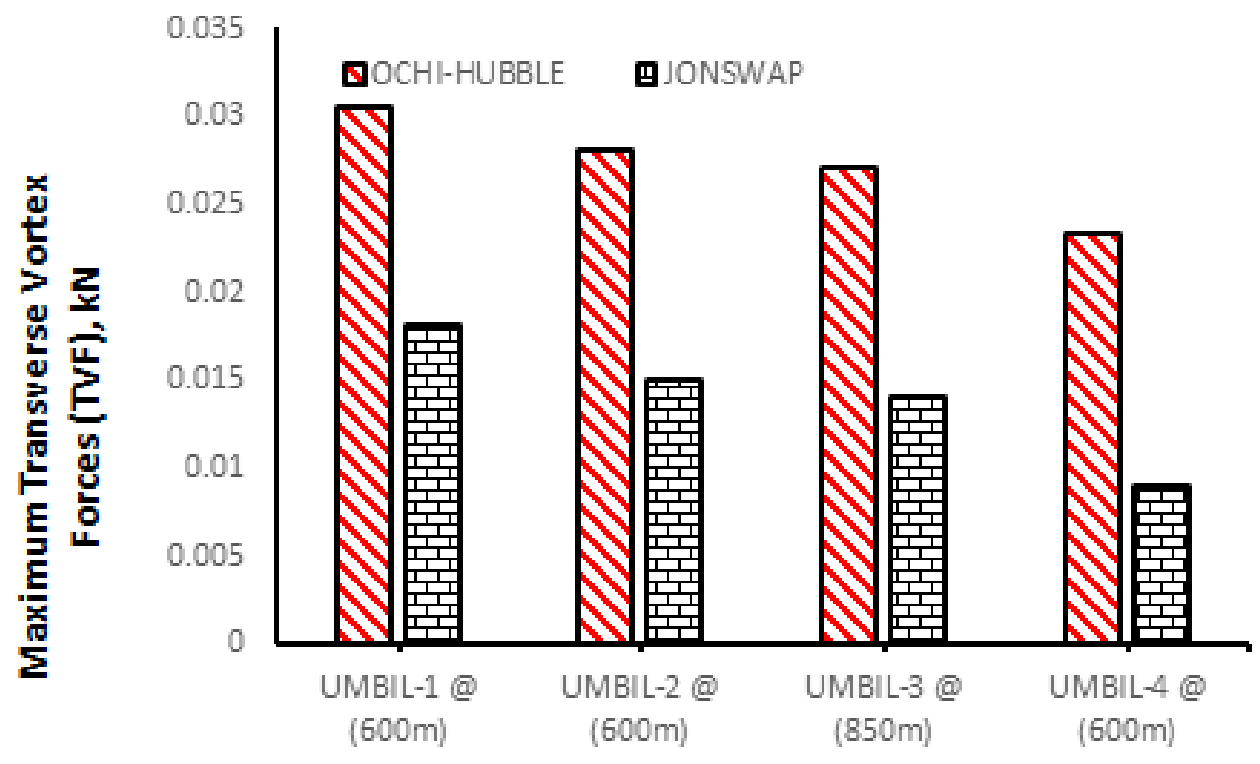

Figure 13. TVF Highest Maxima for Ochi- Hubble and JONSWAP

In addition, Figure 13 equally shows the highest maxima TVF values for Umbilical-1 (600m), Umbilical-2 (600m), Umbilical- $3(850 \mathrm{~m})$, and Umbilical-4 $(600 \mathrm{~m})$ for both wave spectra. It can be clearly seen that the bar chart shows heights in descending order for Ochi Hubble and JONSWAP. Hence, the TVF values decrease steadily from Umbilical-1 through Umbilical-4. From this Figure 13 Umbilical-1 showed the maximum TVF while Umbilical-4 showed the minimum. 


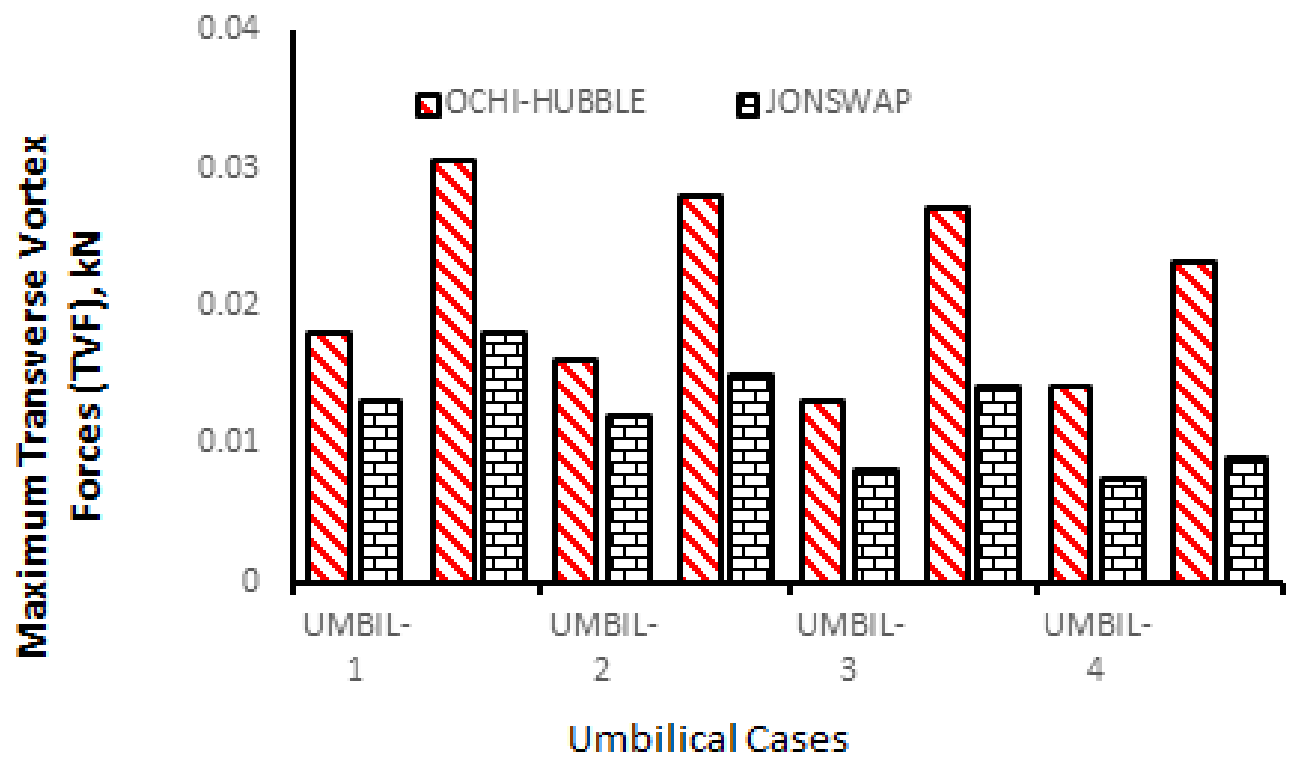

Figure 14. TVF Maxima for Ochi- Hubble and JONSWAP

Considering both wave spectra in the analysis of Umbilicals 1 to 4 , the results for JONSWAP were shown. Umbilical-1, Umbilical-2 and Umbilical-4 at arc length $300 \mathrm{~m}$ and $600 \mathrm{~m}$ gave highest maxima $T V F$ values for $0.013 \mathrm{kN}$ and $0.018 \mathrm{kN} ; 0.012 \mathrm{kN}$ and $0.0149 \mathrm{kN} ; 0.0074 \mathrm{kN}$ and $0.0086 \mathrm{kN}$, respectively. Umbilical-3 at $300 \mathrm{~m}$ and $850 \mathrm{~m}$ gave $0.008 \mathrm{kN}$ and $0.014 \mathrm{kN}$. It can be seen that the $T V F$ values at $600 \mathrm{~m}$ and $850 \mathrm{~m}$ were higher than that of $300 \mathrm{~m}$. Hence, at near- surfaces the deeper the Umbilicals are from the FPSO the higher the TVF. Similarly for the Ochi-Hubble spectra results, Umbilical-1, Umbilical-2 and Umbilical-4 at arc length $300 \mathrm{~m}$ and $600 \mathrm{~m}$ gave highest maxima $T V F$ values for $0.018 \mathrm{kN}$ and $0.0305 \mathrm{kN} ; 0.016 \mathrm{kN}$ and $0.028 \mathrm{kN}$; $0.0141 \mathrm{kN}$ and $0.0232 \mathrm{kN}$, respectively. Umbilical-3 at $300 \mathrm{~m}$ and $850 \mathrm{~m}$ gave $0.013 \mathrm{kN}$ and $0.027 \mathrm{kN}$. Also as the case of $J O N S W A P$, it can be seen that the TVF values at $600 \mathrm{~m}$ and $850 \mathrm{~m}$ were higher than that of $300 \mathrm{~m}$. Hence, at nearsurfaces the deeper the Umbilicals the higher the $T V F$.

The comparison of Figures 12, 13 and Figure 14 reveals that the positioning of the Umbilicals at sea with respect to the current direction and speed at the various wave frequencies is the primary cause of the disparity in TVF values and not the order in which the Umbilicals are numbered in this work. From literature review, the JONSWAP model though it was designed for the North Sea, is used almost anywhere because the bandwidth can be adjusted by changing its peakedness parameter $\gamma$. However, there is little evidence to support its use when the climate is somewhat different from that of North Sea. Ewans et al. [13] and Forristall et al. [14] suggested the use of Gaussian or log-normal distributions. Normally double-peak is associated with Ochi-Hubble spectra while single peak is associated with JONSWAP. For the Ochi-Hubble spectra analysis, the results showed double-peak which was not consistent.
This result clearly shows that the adjustment of the peakedness parameter of the JONSWAP based design to suit Ochi-Hubble environment of Offshore Nigeria is in proper alignment. However, from the results, its precision is not guaranteed since this phenomenon is not applicable to every slope on the graph.

\section{Conclusions}

The dynamic analysis due to Vortex Induced Vibrations on the four Umbilicals gave highest maxima $T V F$ values at the near surfaces (water depths of $300 \mathrm{~m}, 600 \mathrm{~m}$ and $850 \mathrm{~m}$ ) for the Ochi-Hubble wave spectra against the JONSWAP. This clearly shows that using a JONSWAP based umbilical design on an Ochi-Hubble environment like Offshore Nigeria causes higher VIV at the near surfaces and consequently a higher fatigue damage tendency in the future. Based on the findings in this study, design of Umbilicals for installation in Deep-Offshore Nigeria should be based on proper research on the wave spectra of this region (Ochi-Hubble) as adjusting the peakedness of the JONSWAP spectra does not give the desired accuracy and hence has significant consequences on the VIV and Transverse Vortex force values.

\section{Nomenclature}

$\begin{array}{ll}T V F & \text {-Transverse Vortex Force } \\ P M & \text {-Pierson and Moskowitz } \\ G_{o} & \text {-Normalization factor } \\ S D & \text {-Spectral Density } \\ D & \text {-Double } \\ W S & \text {-Wind Sea }\end{array}$




$\begin{array}{ll}W & \text {-Wallops } \\ f & \text { - Frequency }[\mathrm{Hz}] \\ f_{n} & \text { - Normalized frequency } \\ f_{p} & \text {-Peak frequency }[\mathrm{Hz}] \\ g & \text { - Acceleration due to gravity }\left[\mathrm{m} / \mathrm{s}^{2}\right] \\ H_{s} & \text {-Significant wave height }[\mathrm{m}] \\ j & \text { - Integer counter } \\ S_{f} & \text { - Wave spectral density }\left[\mathrm{m}^{2} / \mathrm{Hz}\right] \\ T_{p} & \text {-Peak period [s] } \\ T_{s} & \text {-Average zero up-crossing period }[\mathrm{s}] \\ t & \text {-Time }[\mathrm{s}] \\ u^{*} & \text { - friction wind velocity }[\mathrm{m} / \mathrm{s}] \\ \alpha & \text {-Parameter } \\ \beta & \text {-Wave direction }[\text { deg] } \\ \lambda & \text {-Ochi-Hubble slope parameter }(5 \& 6) \\ \gamma & \text {-Peakedness parameter }(1 \text { to } 7) \\ \Gamma & \text { - Gamma function }\end{array}$

\section{REFERENCES}

[1] Phillips, O.M. (1958); The equilibrium range in the spectrum of wind generated waves. J. Fluid Mech. Vol.4, pp. 426-434.

[2] Pierson, W.J. and Moskowitz, L. (1964); A proposed spectral form for fully developed wind seas based on the similarity of S.A. Kitaigorodskii, J. Geophys. Res., Vol.69, no. 24, pp. 5181-5190.

[3] Hasselmann, K, Barnett, T.P. Bouws, E.Carlson, H Cartwright, D.E. Enke, K. Ewing, J.A, Gienapp, H. Hasselmann, D.E, Kruseman, P. Meerburg, A. Muller, P. Olbers, D.J. Richter, K. Sell, W. \& Walden, H. (1973); Measurements of wind-wave growth and swell decay during the Joint North Sea Wave Project, Deutsche Hydrographische Zeitschrift, Institute Reihe A $\boldsymbol{8}$ (Nr.12), pp 95 .
[4] Toba, Y. (1972); Local balance in the Air-Sea Booundary Process. I. On the growth process of wind waves. $J$. Oceanographic Soc. Japan. Vol.28 pp. 109-121

[5] Phillips, O., M. (1984); Spectral and statistical properties of the equilibrium range in wind generated gravity waves. J. Fluid Mech Vol.156 pp 505-531.

[6] Bretschneider, (1958); Revision in forecasting: Deep and shallow water proc. Int. conf. coastal Eng. ASCE $6^{\text {th }} \mathrm{pp}$. 30-67.

[7] Ochi, M.K., Hubble, E.N. (1976); On six-parameters wave Spectra, Proc. $15^{\text {th }}$ Coastal Eng. Conf. Vol. 1, pp. 301-328.

[8] Massel, S.R. (1996); Ocean Surface Waves, their Physics and Prediction, World Scientific. Advance Series of Ocean Eng. Conf. Vol. 11, pp 491-508

[9] Hasselmann, K., Ross, D.B., M“uller, P. and Sell, W. (1976); A parametric wave prediction model, J. Phys. Oceanogr., Vol. 6, no. 2, pp. 200-228.

[10] Mansour, A., and Ertekin, R., Eds. (2003); Report of Technical Committee I.1 - Environment Proc. 15th ISSC, Vol. 1 .

[11] Quiniou-Ramus, V., Hoche, M.A., Francois, M., Nerzic, R., Ledoux, A., Orsero, M. (2003); Recent Breakthroughs in the Analysis of Total E\&P Angola Block 17 wind/wave/current records and their impact on floating structures design, Proc. XVth Deep Offshore Technology Conf. , DOT, Marseilles.

[12] OrcaFlex 8.4a7 (2003); Orcina Ltd, Daltongate, Ulverston, Cumbria UK.

[13] Ewans, K., Forristall, G.Z., Olagnon, M. and Prevosto, M. (2013); Response sensitivity to swell spectra off West Africa, Proc. 32nd Int. Ocean, Offshore and Arctic Eng. Conf., OMAE pp.2013-11252.

[14] Forristall, G.Z., Ewans, K., Olagnon, M. and Prevosto, M. (2013); The West Africa Swell Project (WASP), Proc. 32nd Int. Ocean, Offshore and Arctic Eng. Conf., OMAE. pp. 2013-11264. 